\title{
Los sistemas de alerta temprana, una herramienta que fortalece la vigilancia epidemiológica en el marco de la seguridad y salud en el trabajo
}

\author{
Víctor Zein Rizo Tello1, ${ }^{2}$ (iD), Astrid Maribel Aguilera Becerra ${ }^{1}$ (D), Laura Ximena Ramírez López
}

\section{RESUMEN}

Introducción: Las enfermedades y los accidentes laborales no solo reducen la productividad y la capacidad de trabajo, sino que también generan altos costos para los trabajadores, empresas, familias, el sistema de salud y la sociedad, motivo por el cual en los últimos años se han convertido en un problema de salud pública que preocupa a las empresas y al gobierno, porque se requiere un enfoque preventivo y anticipado integral.

Objetivo: compilar información actualizada y relevante acerca de los sistemas de alerta temprana que puedan aplicarse en el marco de la vigilancia epidemiológica en la seguridad y salud en el trabajo.

Materiales y métodos: Revisión narrativa de la literatura, a partir de la búsqueda en diferentes bases de datos de artículos publicados durante 2016-2021. Los documentos seleccionados fueron artículos originales, de revisión, guías de manejo, implementación y legislación nacional, que abordan los temas de inteligencia epidemiológica, sistemas de alerta temprana y seguridad y salud ocupacional.

Resultados: Los sistemas de alerta temprana son herramientas que permiten proveer una información oportuna y eficaz que ayuda a los individuos expuestos a tomar decisiones para evitar o reducir el riesgo.

Conclusiones: La implementación de un sistema de alerta temprana en las empresas ayudará a disminuir los tiempos desde la identificación del riesgo hasta la respuesta y, así, con una mayor oportunidad de control, se influirá en la salud de los trabadores, la producción y finanzas de la empresa.

Palabras clave: vigilancia; inteligencia; salud pública; medidas de seguridad; medicina preventiva; medicina del trabajo; enfermedades profesionales; salud laboral.

1 Universidad de Boyacá, Tunja, Colombia.

2 Instituto Nacional de Salud, Bogotá, Colombia.

Autor de correspondencia: Víctor Zein Rizo Tello. Correo electrónico: vzrizo@uniboyaca.edu.co Citar este artículo así:

Rizo Tello VZ, Aguilera Becerra AM, Ramírez López LX. Los sistemas de alerta temprana, una herramienta que fortalece la vigilancia epidemiológica en el marco de la seguridad y salud en el trabajo. Rev Investig Salud Univ Boyacá. 2021;8(1):152-169. https://doi.org/10.24267/23897325.623 


\title{
Early Warning Systems, A Tool That Strengthens Epidemiological Surveillance within the Framework of Occupational Health and Safety
}

\begin{abstract}
Introduction: Occupational diseases and accidents not only reduce productivity and work capacity, but also generate high costs for workers, companies, families, the health system, and society, which is why in recent years they have become a public health problem, which worries companies and the government, and achieving a longer and healthier working life has become increasingly difficult, requiring a comprehensive anticipatory and preventive approach.
\end{abstract}

Objective: Conduct an exploratory review of relevant information about Early Warning Systems that can be applied in the framework of epidemiological surveillance in occupational health and safety.

Materials and methods: Conduct an exploratory review of were carried out in different databases of articles published during 2016-2021. The documents that were selected in their original articles, review, management guides, implementation, and national legislation, which address the issues of epidemiological intelligence, early warning systems and occupational health and safety.

Results: The Early Warning Systems are tools that provide timely and effective information that helps subjects exposed to a threat to make decisions to avoid or reduce the risk.

Conclusions: The implementation of an early warning system in companies will help reduce the times from risk identification to response and thus have a greater opportunity for control, thus impacting the health of workers, the production, and finances of the company.

Keywords: surveillance; intelligence; public health; security measures; preventive medicine; occupational medicine; occupational diseases; occupational health. 


\title{
Os sistemas de alerta precoce, um instrumento para reforçar a vigilância epidemiológica no âmbito da saúde e segurança no trabalho
}

\begin{abstract}
RESUMO
Introdução: As doenças e acidentes de trabalho não só reduzem a produtividade e a capacidade de trabalho, como também geram custos elevados para os trabalhadores, as empresas, as famílias, o sistema de saúde e a sociedade, razão pela qual nos últimos anos se tornaram um problema de saúde pública que preocupa as empresas e o governo, porque é necessária uma abordagem preventiva, antecipada e integral.
\end{abstract}

Objetivo: Compilar informação atualizada e relevante sobre os sistemas de alerta precoce que podem ser aplicados no âmbito da vigilância epidemiológica em segurança e saúde ocupacional.

Materiais e métodos: Revisão narrativa da literatura, a partir da pesquisa em diferentes bases de dados de artigos publicados durante 2016-2021. Os documentos selecionados foram artigos originais, artigos de revisão, orientações de gestão, execução e legislação nacional, abordando os tópicos da inteligência epidemiológica, sistemas de alerta precoce e saúde e segurança no trabalho.

Resultados: Os sistemas de alerta precoce são ferramentas que fornecem informações oportunas e eficazes para ajudar os indivíduos expostos a tomar decisões para evitar o reduzir o risco.

Conclusões: A aplicação de um sistema de alerta precoce nas empresas ajudará a reduzir o tempo desde a identificação do risco até a resposta e, assim, com uma maior probabilidade de controle, se influenciará na saúde dos trabalhadores, na produção e nas finanças da empresa.

Palavras-chave: vigilância; inteligência; saúde pública; medidas de segurança; medicina preventiva; medicina ocupacional; doenças profissionais; saúde ocupacional. 


\section{INTRODUCCIÓN}

La gran mayoría de las enfermedades profesionales no son curables o tienen un tratamiento difícil y de larga duración (1). Estas condiciones reducen la productividad y la capacidad de trabajo, pero con el adicional de generar altos costos para los trabajadores, sus familias, las empresas y la sociedad, los cuales son sumados a los costos intangibles también conocidos como costos indirectos, pues el sufrimiento humano no puede medirse de ninguna manera (2-5).

La incidencia de enfermedades relacionadas con la salud mental, los riesgos psicosociales y la tensión relacionada con el trabajo han aumentado, al mismo tiempo que lo han hecho las enfermedades crónicas no transmisibles originadas en el entorno laboral, por un inadecuado control de los factores de riesgo cardiovascular, como falta de actividad física, obesidad, dislipidemia o fumar, que a futuro representarán en el trabajador enfermedades como hipertensión arterial, infarto agudo de miocardio y accidente cerebrovascular. Del mismo modo, los hábitos alimenticios derivados de los horarios laborales redundan en trastornos digestivos o enfermedades metabólicas $(2,6)$.

Según estimaciones de la Organización Internacional del Trabajo, las enfermedades y las lesiones profesionales provocan una pérdida anual del
$4 \%$ del producto interno bruto mundial, lo que motiva que se mejoren la salud y seguridad en el trabajo dentro de las organizaciones y se incentive la investigación en la descripción de la historia natural de la enfermedad y los factores de riesgo, de modo tal que se tomen decisiones en relación con su prevención, detección precoz y tratamiento $(5,7,8)$.

En general, se acepta que el entorno laboral no debería presentar un riesgo de lesiones o enfermedades; sin embargo, en los últimos años, las enfermedades laborales se han convertido en un problema de salud pública y han creado la necesidad de trabajar con un enfoque preventivo integral. Por tal motivo, la mayoría de las empresas han desarrollado e implementado sistemas de registro de enfermedades profesionales, a fin de lograr una adecuada caracterización e implementación de actividades de educación y promoción de la salud e intervención sobre ellas (9).

Las enfermedades laborales se explican a partir de la tríada epidemiológica agente, ambiente $y$ huésped, de su historia natural y del modelo multicausal. Así es como, desde hace mucho tiempo, las acciones de prevención intervienen los factores de riesgo de forma separada, no integran las interacciones aditivas ni los factores personales $y$ familiares que podrían ser variables de confusión, porque ocultarían la posibilidad de hallar la verdadera etiología (10-12). 
La epidemiología, en el marco de la seguridad y salud en el trabajo (SST), aún tiene mucho por avanzar, pues se ha dedicado a captar datos de forma pasiva y a encontrar factores de riesgo en las evaluaciones de ingreso, periódicas o de egreso, para llevar un registro histórico (13); pero no para tomar decisiones oportunas. Lo anterior permite plantear la necesidad de que la vigilancia epidemiológica pasiva descriptiva evolucione a una vigilancia activa-analítica e innovadora, sin perder la integralidad de ambas, a fin de configurar lo que se conoce como inteligencia epidemiológica, con la que se quiere anticipar la respuesta al riesgo y disminuir el impacto en la salud de los empleados, los costos para la empresa, las administradoras de riesgos laborales, la sociedad y la familia. El objetivo de este artículo es compilar información actualizada y relevante acerca de los sistemas de alerta temprana (SAT), que puedan aplicarse en el marco de la vigilancia epidemiológica en la SST.

\section{METODOLOGÍA}

Para la elaboración del artículo se llevó a cabo una de revisión narrativa de la literatura, teniendo en cuenta como palabras clave las validadas en los Descriptores en Ciencias de la Salud, en diferentes combinaciones, en las bases de datos Medline con la ayuda del buscador PubMed (que se complementó con LILACS para tener resultados de países de Latinoamérica y el Caribe),
SciELO, Biblioteca Virtual en Salud, Redalyc, Directory of Open Access Journals; además del portal de Scimago, para identificar las revistas de mayor impacto en epidemiología, SST o medicina laboral. Finalmente, hubo una pesquisa en bola de nieve en buscadores genéricos de internet. En la estrategia de búsqueda se utilizaron los filtros de título y resumen y se conjugaron según los conectores boleanos para optimizarla.

Los criterios de selección fueron: artículos originales, de revisión, guías de manejo e implementación y legislación nacional, que abordan los temas de inteligencia epidemiológica, sistemas de alerta temprana y seguridad y salud ocupacional. Se tuvo en cuenta para la selección que hayan sido publicados en los últimos cinco años, debido a su relevancia teórica y conceptual.

Los criterios de exclusión fueron: artículos en idiomas diferentes al español y al inglés. Se captaron 55 artículos, de los cuales se seleccionaron 50 que cumplieron con los criterios de inclusión.

\section{RESULTADOS}

El riesgo se define como la incertidumbre que tienen las empresas en el momento de ejecutar las acciones para lograr sus objetivos, las cuales están influidas por factores externos (peligros o amenazas) e internos (vulnerabilidad o susceptibilidad). Cuando se habla de SST dentro de las 
organizaciones, no es negociable el hecho de tener que hablar de riesgo, porque en todas las empresas existen y cada una lo gestiona a su medida. Este concepto es tan amplio que incluye el evento cuya ocurrencia se quiere cambiar, los factores causales y los efectos generados cuando el evento finalmente ocurre (14-16).

La epidemiología es importante en la SST, pues su finalidad es estudiar la distribución y los determinantes del estado de salud-enfermedad y su aplicación al control de problemas de salud (11). En el entorno laboral, el Ministerio del Trabajo, a través del Decreto 1072 del 2015, hace referencia a la epidemiología como la herramienta para vigilar la salud en el trabajo que recopila, analiza, interpreta, difunde continua y sistemáticamente datos a efectos de la prevención y ser el sostén para planificar, ejecutar y valorar los programas de SST (17).

La vigilancia epidemiológica no únicamente usa la estadística y reporta cifras, sino que predice y gestiona las situaciones que afectan el bienestar de las personas, desde los factores de riesgo, a través de la inteligencia epidemiológica, a partir de la identificación, verificación, notificación, confirmación, investigación y respuesta temprana, que garantice la reducción de los daños y su impacto dentro de una organización (18-20).
En este artículo se quiere proponer el uso de la inteligencia epidemiológica como la integración de la "vigilancia basada en indicadores", es decir, la recolección, análisis e interpretación de datos estructurados desde los sistemas de vigilancia existentes y la "vigilancia basada en eventos", que consiste en la captura, filtrado y verificación de información proveniente de fuentes oficiales y no oficiales, pero que tiene la capacidad de aprender de la experiencia y adaptarse de acuerdo con los datos actuales, de manera que se vuelvan cada vez más sensibles en percibir y responder a la situación y al ambiente $(18,21,22)$.

Lo anterior hace referencia a los SAT, y que en este artículo quieren ser una propuesta para su implementación y ejecución en el marco de la SST en las empresas. Los SAT son:

[...] herramientas que permiten proveer una información oportuna y eficaz a través de instituciones técnicas, científicas y comunitarias, por medio de elementos, que permiten a los individuos expuestos a una amenaza latente, la toma de decisiones para evitar o reducir su riesgo y su preparación para que puedan brindar una adecuada respuesta teniendo en cuenta sus capacidades. (23)

En otras palabras, el fin último del SAT es integrar a la comunidad y convertirla en un componente importante y activo de la gestión que, junto con las demás herramientas, pueda ejecutar acciones 
de forma rápida, eficiente, que aumente la oportunidad de control (24).

El término SAT ha sido ampliamente utilizado en la gestión de riesgo de desastres, porque ha mostrado ser útil en la reducción de la vulnerabilidad y la mortalidad, al tiempo que ha mejorado la resiliencia de las comunidades (25). Un ejemplo de ello es Colombia, un país que ha tenido que priorizar el fortalecimiento de los SAT, debido a su variabilidad climática y los desafíos inherentes al cambio climático y los peligros naturales (26).

Implementar y ejecutar los SAT en las empresas y hacer parte de estos a los trabajadores, puede influir significativamente en los accidentes en el trabajo y la enfermedad laboral, cuando se interioriza que su eficacia para reducir la exposición a los peligros está sujeta a la competencia de las personas para entender y responder a una situación determinada.

Históricamente, los SAT se han centrado en infraestructura y tecnología, pero no han fortalecido la estructura de trabajo y las cadenas de mando, y ello ha ocasionado la ausencia de un trabajo articulado con la comunidad según los cuatro elementos del sistema: conocimiento de riesgos, monitoreo, capacidad de comunicación y respuesta, lo que ha mostrado que la comunidad tenga reacciones y respuestas inapropiadas durante los desastres $(27,28)$.
A continuación, se contextualiza el SAT, teniendo en cuenta los conceptos del Reglamento Sanitario Internacional y el modelo del Sistema de Gestión del Riesgo, correspondiente a la norma ISO31000, y su aplicación epidemiológica en el marco de la SST (29).

Los SAT deben ser creados con bases sólidas. Así, la empresa debe contar con una adecuada planeación y preparación, que garantice la existencia de cada uno de los procesos y procedimientos que documenten las actividades de vigilancia epidemiológica laboral y los requerimientos de la legislación local, armonizados con el mapa de procesos de la organización (29).

La figura 1 es la propuesta del sistema de gestión del riesgo de la norma ISO31000 que adapta el SAT a la SST, y cuya principal característica es la flexibilidad a la adaptación según el tamaño de la empresa, pues dependiendo de este y de la actividad económica, serán las capacidades básicas para la gestión (30).

El modelo inicia con el establecimiento del contexto de la organización, el cual comprende dos escenarios: 1) el contexto externo, que involucra requisitos legales, así como reglamentación del ambiente social, cultural, político, económico, tecnológico, y todo lo demás que externamente pueda influir en la gestión del riesgo. 2) El contexto interno, que comprende la cultura de la 
Figura 1. Sistema de gestión del riesgo adaptado a la seguridad y salud en el trabajo
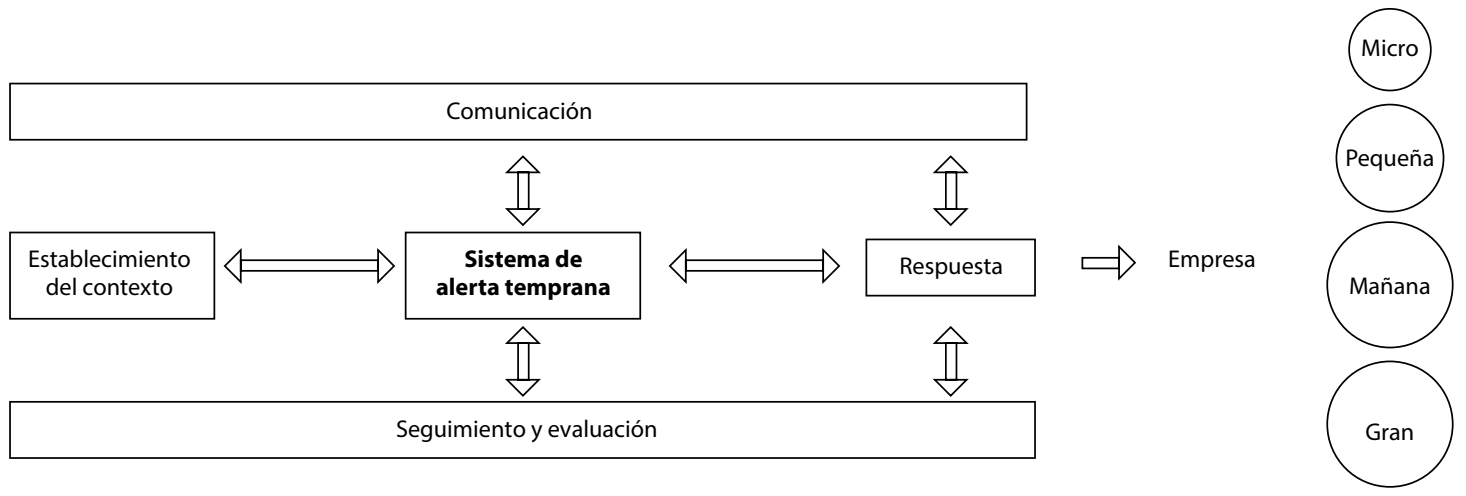

Fuente: tomado de la norma ISO31000 y adaptada por los autores de este artículo.

organización, los procesos, la estructura y la estrategia organizacional (16).

La siguiente etapa es la identificación del riesgo, que integra herramientas y actividades para encontrarlo, reconocerlo y describirlo a partir de la detección de las fuentes, las causas, las áreas de impacto y sus consecuencias. Posteriormente, un grupo de expertos debe realizar el análisis y la valoración del riesgo, haciendo hincapié en las amenazas y vulnerabilidades, para que finalmente el riesgo se pueda cuantificar y clasificar $y$, a partir de ahí, definir las acciones de respuesta proporcionales (16).

EI SAT debe ser una estructura integradora, entre la gestión del riesgo y la vigilancia epidemiológica, que aumente la eficiencia de la gestión. Debe trabajar permanentemente, y tener un gestor con disponibilidad completa los siete días de la semana, encargado de dirigir y administrar la gestión de situaciones y articularla con las áreas de la empresa correspondiente.

Así mismo, el SAT es un modelo abierto de entrada de señales de alarma y peligros que deben pasar por un filtro de verificación, organización y generación de información, a efectos de ser presentado a los interesados y tomadores de decisiones de forma organizada para definir la respuesta adecuada. La figura 2 es una propuesta que evidencia la transversalidad de los SAT en el marco de la SST. 
Figura 2. Sistema de alerta temprana en el marco de la seguridad y salud en el trabajo

\section{Entrada}

Registro de incapacidades.

- Base de auto reporte del estado

de salud del trabajador.

- Atención medica laboral en la empresa.

- Rumores.

- Sensores laborales (compañero de trabajo, coordinador, vendedor de la cafetería, etc.).

- Correo electrónico de vigilancia e

salud laboral.

- Teléfono para el reporte de eventos de

salud laboral.

- Sensores familiares.

- Aplicaciones móviles.
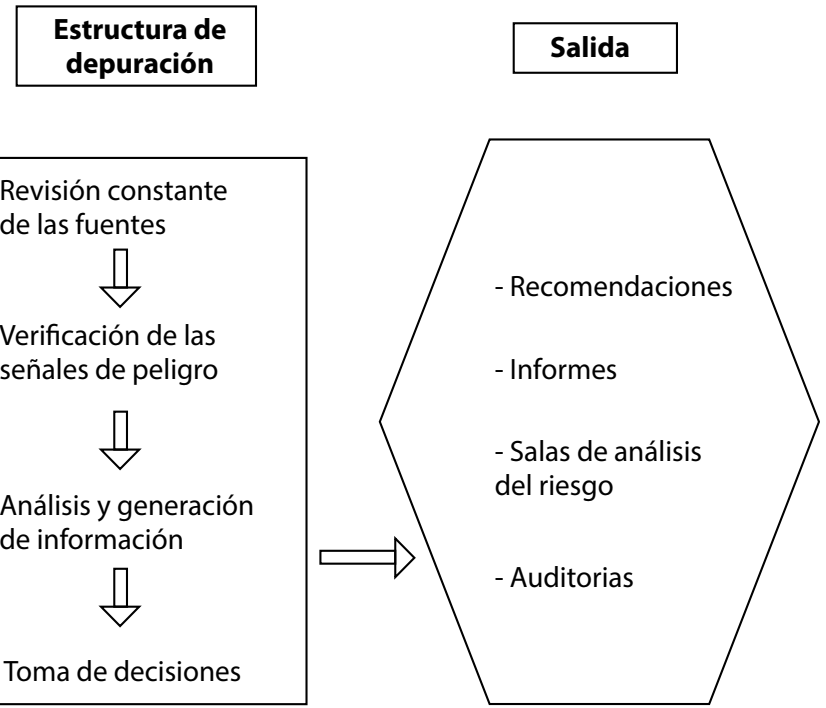

Fuente: tomado de los lineamientos para la vigilancia y control de los eventos de interés en salud pública 2021. Estrategia basada en casos predefinidos y del Grupo de Gestión del Riesgo Respuesta Inmediata en Salud Pública del Centro de Operaciones de Emergencias en Salud Pública del Instituto Nacional de Salud y adaptada por los autores de este artículo.

Como cualquier sistema de salud, la gestión del riesgo impacta en las comunidades cuando se logran prevenir e identificar rápidamente las enfermedades y los accidentes laborales. En este instante, es fundamental poner en práctica las estrategias de la vigilancia innovadora comunitaria, usadas en Colombia en el contexto de la salud pública y la contingencia de la pandemia de la covid-19 $(31,32)$, como parte de las estrategias de vigilancia activa que ayudan al SAT a gestionar el riesgo: captación y verificación de rumores, sensores laborales, correo electrónico, aplicaciones móviles, línea telefónica y los sensores familiares.

La captación y verificación de rumores debe darse en el contexto que beneficie al SAT; por lo cual su gestión debe ser responsable y tener un objetivo claro. Se trata de identificar alertas o peligros a partir de comentarios de pasillo, en espacios de esparcimiento y descanso, los cuales son canalizados a través del SAT para iniciar el trámite correspondiente. 
Los sensores laborales (son el equivalente del sensor comunitario en salud pública) hacen parte de las estrategias orientadas a la definición e identificación de situaciones, factores de riesgo o determinantes en los empleados que pueden afectar su salud y calidad de vida. En general, se trata de una red de personas o áreas de la empresa que, luego de recibir una capacitación basada en manejo y control y con un alto interés, se convierte en un apoyo para realizar actividades de vigilancia de los eventos y circunstancias priorizadas, teniendo en cuenta que deben ser identificados de forma mayoritaria por los trabajadores, como resultado de un ejercicio de diagnóstico local participativo y, además, ser susceptible de intervención por parte del mismo grupo laboral o de la empresa $(33,34)$.

De la misma manera, esta red debe tener la capacidad de identificar otras situaciones que no estén priorizadas, que sean de novo o de carácter individual en cualquier trabajador. Esas alertas captadas deben ser dirigidas al SAT para la gestión correspondiente.

El uso de un correo electrónico, aplicaciones móviles y una línea de atención telefónica, que sean atendidos todo el día todos los días de la semana, para que el trabajador pueda informar sobre su estado de salud, antes de asistir a una consulta médica y después de esta, de manera que el equipo de SST esté al tanto y pueda anticiparse a la situación (tal es el caso de enfermedades transmisibles respiratorias o gastrointestinales que pueden estar relacionadas con alta contagiosidad y ausentismo), a partir de la vigilancia sindrómica activa inteligente (35).

Finalmente, existe el escenario en que el empleado no quiera faltar a sus actividades o no quiera un descuento en su salario por concepto de incapacidad, motivo por el cual no avisa de su alteración de salud o evita que ello sea percibido por sus compañeros y superiores, por lo cual es importante implementar el sensor familiar, donde un integrante de su núcleo puede dar aviso del estado de salud del trabajador para que sea abordado en la empresa por parte del grupo encargado.

Entonces, ¿qué se debe vigilar en el SAT? Debe vigilar todo aquel riesgo o peligro que pueda causar al trabajador un accidente o una enfermedad laboral y que se relaciona a continuación: enfermedad general, enfermedad laboral (8), situaciones relacionadas con el riesgo ergonómico (36), situaciones relacionadas con los accidentes laborales (37), factores de riesgo para enfermedad laboral y accidente de trabajo (37-39), incapacidades, alertas, brotes, epidemias o emergencias en salud, originadas dentro o fuera de la empresa, que puedan afectar la salud del trabajador (40-42). 
Además, es importante que, a partir de la evaluación de ingreso de cada trabajador, que comprende las características sociales, económicas, culturales y la identificación del estado previo de salud, sus factores de riesgo y las actividades que va a desempeñar en la empresa, se puedan establecer poblaciones de vigilancia especial. Por ejemplo: trabajadores con alguna discapacidad previo a su ingreso, población migrante en situación legal, indígenas, afrocolombianos, mineros o reinsertados a la sociedad, de tal manera que el SAT para estos grupos pueda ser más sensible, captando señales de alerta, procesando y dando respuesta $(31,32)$.

Dentro de las actividades del SAT se deben revisar las fuentes de información epidemiológica, verificar las señales de alarma o peligro y analizar la información que, todo ello en conjunto, es considerado monitoreo, el cual se encuentra representado en la figura 2, como la estructura de depuración del sistema. En esta etapa es de gran relevancia que la empresa establezca un perfil epidemiológico y de riesgo (línea de base) y a partir de este se realice un constante seguimiento de la frecuencia de los factores de riesgo encontrados o eventos ocurridos en la empresa que afecten la salud de los factores de forma individual y grupal. El modelo pierde sentido si la persona (gestor del SAT) que está al tanto de este proceso no tiene el conocimiento y entrenamiento en salud, prevención, gestión del riesgo, análisis de información epidemiológica en el contexto de la SST que pueda hallar las asociaciones y la causalidad, los comportamientos inusuales en el reporte de accidentes y enfermedad laboral y que entienda la importancia de la buena comunicación, trabajo en equipo y la velocidad en la gestión de la información para responder de forma adecuada. Este individuo tiene la responsabilidad de tomar decisiones y definir la necesidad de convocar grupos interdisciplinarios para analizar, evaluar y dar respuesta.

EI SAT debe ser el centro de la gestión de la información relacionada con los eventos ocurridos en el ámbito laboral. Es importante que exista una gran responsabilidad, compromiso y una formación ética que mantenga la confidencialidad de la información y ejerza la imparcialidad en el momento de ejecutar las actividades. Deben establecerse los requisitos mínimos necesarios para que el trabajo se encuentre dentro de los parámetros considerados éticos, los cuales deben estar claramente establecidos y justificados, prácticos e implementables, y deben estar articulados con el componente legal que garantice la seguridad de la información para que no se comprometa la funcionalidad e imagen de la empresa (43-48).

Finalmente, el SAT tiene una importante función en la emisión de la información de forma oportuna; por tal motivo, cada etapa del modelo debe complementarse y acompañarse con habilidades en la 
comunicación del riesgo, que abarque el establecimiento de los componentes de cada mensaje clave que se deba emitir (caracterización del individuo y de la situación, tipo de población afectada, área de trabajo al que pertenece), la estructura a partir de la cual va a fluir la información desde el trabajador hasta el grupo de SST y cómo desde este grupo a los encargados de tomar decisiones $(49,50)$.

\section{CONCLUSIÓN}

Se resalta la importancia de implementar las estrategias de vigilancia innovadora, en especial el SAT, para poder disminuir los tiempos desde la identificación del riesgo hasta la respuesta, y así tener mayor oportunidad de control. Ello influirá en la salud de los trabajadores, en la producción y en las finanzas de la empresa. Se recomienda propiciar que evolucionen las actividades del grupo de SST de las empresas, para que esté al tanto de los riesgos y no espere a su reporte para realizar la caracterización, evaluación y mejoras, sino que este equipo tenga como meta la anticipación, lo que requiere un fortalecimiento continuo de capacidades del personal y de la institución. El SAT es un modelo de ayuda y debe ser usado de manera imparcial para mejorar la seguridad y la salud de los empleados, no como una herramienta que favorezca el juicio y el señalamiento hacia los empleados.

\section{AGRADECIMIENTOS}

A la Universidad de Boyacá, por brindar el espacio académico de relación docente- estudiante, para la producción de literatura científica que ayuda a fortalecer las acciones de la comunidad académica mundial, y al Instituto Nacional de Salud, por sus aportes a la gestión del riesgo en salud pública del país.

\section{FINANCIACIÓN}

Los costos generados en la elaboración y publicación de este trabajo son asumidos por los autores.

\section{CONFLICTOS DE INTERESES}

Los autores declaramos que no tenemos ningún vínculo comercial, financiero con alguna empresa y no tenemos conflictos de intereses, diferente a generar información académica que impacte en la comunidad científica, en beneficio de los empleados y las empresas.

\section{REFERENCIAS}

1. Schulte PA, Pandalai S, Wulsin V, Chun H. Interaction of occupational and personal risk factors in workforce health and safety. Am J Public Health (1971). 2012;102(3):434-48. https://doi.org/10.2105/AJPH.2011.300249 
2. International Labour Organization. Safety and health at the heart of the future of work: building on 100 years of experience [internet]. Geneva: autor; 2019. Disponible en: https:// www.ilo.org/safework/events/safeday/ WCMS_686645/lang--en/index.htm

3. Davoodi S, Haghighi KS, Kalhori SRN, Hosseini NS, Mohammadzadeh Z, Safdari R. Occupational disease registries-characteristics and experiences. Acta Inform Med. 2017;25(2):136-40. https://doi.org/10.5455/ aim.2017.25.136-140

4. Lu Y, Yan H, Zhang L, Liu J. A Comparative study on the prediction of occupational diseases in China with hybrid algorithm combing models. Comput Math Methods Med. 2019 Sep 29;2019:1-10. https://doi. org/10.1155/2019/8159506

5. BaDun M. Costs of occupational injuries and illnesses in Croatia. Arhiv za Higijenu Rada i Toksikologiju. 2017 Mar;68(1):66-73. https:// doi.org/10.1515/aiht-2017-68-2899

6. Driscoll T. Global and regional burden of disease and injury in 2016 arising from occupational exposures: a systematic analysis for the Global Burden of Disease Study 2016. Occup Environ Med. 2019;77(142):133-41. https://doi. org/10.1136/oemed-2019-106008
7. Boschman JS, Brand T, Frings-Dresen MHW, van der Molen HF. Improving the assessment of occupational diseases by occupational physicians. Occup Med. 2017 Jan 1;67(1):139. https://doi.org/10.1093/occmed/kqw149

8. Rushton L. The global burden of occupational disease. Curr Environ Health Rep. 2017;4(3):340-8. https://doi.org/10.1007/ s40572-017-0151-2

9. Loeppke R, Boldrighini J, Stutts C, Perkins C, Russi M, Ording J, et al. Interaction of health care worker health and safety and patient health and safety in the US Health Care System: recommendations from the 2016 Summit. J Occup Environ Med. 2017;59(8):803-13. https://doi.org/10.1097/ JOM.0000000000001100

10. Organización Panamericana de la Salud. Módulos de principios de epidemiología para el control de enfermedades (MOPECE). Módulo: Salud y enfermedad en la población. Vol. 2. Washington, D. C.: Biblioteca de la OPS; 2019.

11. Abdalla S, Apramian SS, Cantley LF, Cullen MR. Occupation and risk for injuries. En: The World Bank, editor. Disease control priorities. 3. ${ }^{\mathrm{a}} \mathrm{ed}$. Vol. 7: Injury prevention and environmental health. Washington: The World Bank; 2017. p. 
97-132. https://doi.org/10.1596/978-1-46480522-6_ch6

12. Viviana D, Vaca B, Parra DC, Joaquín $C$, Dihigo $\mathrm{G}$, Yoel I, et al. Elaboración de un protocolo para la vigilancia de la salud laboral. Rev Méd Electron [internet]. 2017 Jun 5 [citado 2021 abr 16];39(2):188-99. Disponible en: https://www.medigraphic.com/cgi-bin/new/ resumenl.cgi? IDARTICULO $=72430$

13. Gómez-Rivadeneira A. Marco conceptual y legal de la gestión del riesgo en salud en Colombia. Monitor Estratégico [internet]. 2014;(5):4-11. Disponible en: https://www. researchgate.net/publication/330838738_ Marco_conceptual_y_legal_de_la_gestion_ del_riesgo_en_salud_en_Colombia

14. ISOTools. ¿En qué consiste el Sistema de Gestión de la Seguridad y Salud en el Trabajo (SG-SST)? [internet]. Vol. 2020. 2016. Disponible en: https://www.isotools. org/2016/09/06/consiste-sistema-gestion-laseguridad-salud-trabajo-sg-sst/

15. Icontec. Norma técnica ISO 31000 de 2009 [internet]. 2011;1-34. Disponible en: https://www.pdfprof.com/PDF_Image. php?idt $=43518 \& \mathrm{t}=16$
16. Decreto 1072 de 2015, por medio del cual se expide el decreto único reglamentario del sector trabajo [internet]. Ministerio del Trabajo de Colombia; 2015. Disponible en: https:// www.mintrabajo.gov.co/documents/20147/0/ DUR + Sector + Trabajo + Actualizado $+a+15$ + de + abril + +de+2016. pdf/a32b1dcf-7a4e8a37-ac16-c121928719c8

17. Ministerio de Sanidad, Consumo y Bienestar Social de España. ¿Qué es la inteligencia epidemiológica? [internet]. 2012. Disponible en: https://www.mscbs. gob.es/profesionales/saludPublica/ccayes/ inteligenciaepidemiologica/inteEpid. htm\#: : text=Se define como inteligencia epidemiológica,amenaza para la salud pública

18. Kaiser R, Coulombier D, Baldari M, Morgan $D$, Paquet $C$. What is epidemic intelligence, and how is it being improved in Europe? Euro Surveillance: bulletin européen sur les maladies transmissibles. 2006;11(5). https:// doi.org/10.2807/esw.11.05.02892-en

19. Ibrahim S. A comprehensive review on intelligent surveillance systems. Commun Sci Technol [internet]. 2016 May 16;1(1):7-14. https://doi.org/10.21924/cst.1.1.2016.7

20. Belciug S, Gorunescu F. Era of intelligent systems in healthcare. En: Intelligent systems 
reference library. Springer Science and Business Media Deutschland; 2020. p. 1-55. https://doi.org/10.1007/978-3-030-14354-1_1

21. Goniewicz K, Burkle FM. Disaster early warning systems: the potential role and limitations of emerging text and data messaging mitigation capabilities. Disaster Mednd Public Health Prep. 2019 Aug 1;13(4):709-12. https://doi. org/10.1017/dmp.2018.171

22. Ministerio de Educación de Panamá, Unesco. Manual sistemas de alerta temprana [internet]. Ciudad de Panamá: Unesco; 2011. Disponible en: http://www.unesco.org/new/fileadmin/ MULTIMEDIA/FIELD/San-Jose/pdf/Panama MANUAL INFORMATIVO.pdf

23. Unidad Nacional para la Gestión del Riesgo de Desastres. Guía para la implementación de sistemas de alerta temprana [internet]. Vol. 9. Bogotá; 2016. p. 1-33. Disponible en: https:// repositorio.gestiondelriesgo.gov.co/bitstream/ handle/20.500.11762/18505/VOL-9-guiapara-la-implementacion-de sistemas-de-aletatemprana. .pdf? sequence $=18$ \&isAllowed $=y$

24. Khankeh HR, Hosseini SH, Farrokhi M, Hosseini MA, Amanat N. Early warning system models and components in emergency and disaster: a systematic literature review protocol. Systemat
Rev. 2019;8:315. https://doi.org/10.1186/ s13643-019-1211-5

25. Casanueva A, Burgstall A, Kotlarski S, Messeri $A$, Morabito $M$, Flouris $A D$, et al. Overview of existing heat-health warning systems in Europe. Int J Environ Res Public Health. 2019;16(15):2657. https://doi.org/10.3390/ ijerph16152657

26. Macherera M, Chimbari MJ. A review of studies on community based early warning systems. J Disaster Risk Stud. 2016 Apr 19;8(1):206. https://doi.org/10.4102/jamba.v8i1.206

27. Sufri S, Dwirahmadi F, Phung D, Rutherford S. A systematic review of Community Engagement (CE) in Disaster Early Warning Systems (EWSs). Prog Disaster Sci. 2020;5:100058. https://doi. org/10.1016/j.pdisas.2019.100058

28. Organización Mundial de la Salud. Reglamento Sanitario Internacional (2005) [internet]. 2. ${ }^{\text {a }}$ ed. Ginebra; 2008 [citado 2021 Mar 21]. Disponible en: https://www.who.int/ ihr/IHR_2005_es.pdf

29. Decreto $957 / 2019$ del 5 de junio, por el cual se adiciona el capítulo 13 al Título 1 de la Parte 2 del del Libro 2 del Decreto 1074 de 2015, Decreto Único del Sector Comercio, Industria y Turismo y se reglamenta el artículo $2^{\circ}$ de la Ley 
590 de 2000, modificado por el artículo 43 de la Ley 1450 de 2011 [internet]. Ministerio de Comercio, Industria y Turismo. [Citado 2021 Apr 10]. p. 1-7. Disponible en: https://www. mincit.gov.co/normatividad/decretos/2019/ decreto-957-por-el-cual-se-adiciona-elcapitulo-13

30. Instituto Nacional de Salud de Colombia. Lineamientos de vigilancia y control en salud pública: estrategiabasadaen casos predefinidos 2021 [internet]. Bogotá: Instituto Nacional de Salud de Colombia; 2021. Disponible en: http://www.ins.gov.co/Direcciones/Vigilancia/ Lineamientosydocumentos/Lineamientos 2021.pdf

31. Instituto Nacional de Salud. Instructivo para la vigilancia en salud pública intensificada de infección respiratoria aguda asociada al nuevo coronavirus 2019 (COVID-19) [internet]. Bogotá; 2020. Disponible en: https://www. ins.gov.co/Paginas/BibliotecaDigital/covidvigilancia.aspx

32. Secretaría Distrital de Salud. Ficha técnica: Subsistema Vigilancia Comunitaria en Salud Pública [Internet]. Bogotá; 2020. Disponible en: http://www.saludcapital.gov.co/Su_VSP/Vigil_ Comunitaria/Ficha_Tec_Vig_Comunitaria.pdf
33. Ministerio de Salud y Protección Social, Organización Mundial de la Salud, Organización Mundial de la Salud Sinergias. Propuesta metodológica para la operación de la Estrategia de Vigilancia en Salud Pública con base comunitaria [internet]. Bogotá; 2014. Disponible en: https://www.minsalud. gov.co/sites/rid/Lists/BibliotecaDigital/ RIDE/VS/ED/VSP/Propuesta-operacionestrategia-vigilancia-salud-publica-con-basecomunitaria.pdf

34. Jia P, Yang S. Early warning of epidemics: towards a national intelligent syndromic surveillance system (NISSS) in China. BMJ Global Health. 2020 Oct 26;5(10):2925. https://doi.org/10.1136/bmjgh-2020-002925

35. Johannes Andreas W, Johanssons E. Observational methods for assessing ergonomic risks for work-related musculoskeletal disorders: a scoping review. Rev Cienc Salud. 2017 Nov 24;16(8):1-31. https://doi.org/10.12804/revistas.urosario. edu.co/revsalud/a.6840

36. González A, Bonilla-Santos J, Quintero M, Reyes C, Chavarro A. Análisis de las causas y consecuencias de los accidentes laborales ocurridos en dos proyectos de construcción. Rev Ing Construc. 2016 Apr 
1;31(1):5-16. https://doi.org/10.4067/S071850732016000100001

37. Cárdenas MM, Cáceres-del-Carpio J, Mejía CR. Factores de riesgo y causas de lesión en los accidentes laborales de ocho provincias peruanas. Rev Cub Investig Bioméd [internet]. 2020 Sep 1 [citado 2021 Apr 16];39:1-4. Disponible en: http://www.revibiomedica.sld. cu/index.php/ibi/article/view/595

38. Amissah J, Badu E, Agyei-Baffour P, Nakua EK, Mensah I. Predisposing factors influencing occupational injury among frontline building construction workers in Ghana. BMC Res Notes. 2019 Nov 6;12(1):2-8. https://doi. org/10.1186/s13104-019-4744-8

39. Madhav N, Oppenheim B, Gallivan M, Mulembakani P, Rubin E, Wolfe N. Pandemics: risks, impacts, and mitigation. En: Madhav N, Oppenheim B, Gallivan M, Mulembakani $P$, Rubin $E$, Wolfe $N$, editores. Disease control priorities. 3. ${ }^{a}$ ed. Vol. 9. Washington: The World Bank; 2017. https://doi.org/10.1596/978-14648-0527-1_ch17

40. Seeger MW, Pechta LE, Price SM, Lubell KM, Rose DA, Sapru S, et al. A conceptual model for evaluating emergency risk communication in public health. Health Security. 2018 May 1;16(3):193203. https://doi.org/10.1089/hs.2018.0020
41. Khan $Y$, O'Sullivan T, Brown A, Tracey S, Gibson J, Généreux $M$, et al. Public health emergency preparedness: a framework to promote resilience. BMC Public Health. 2018 Dec 5;18(1):1-16. https://doi.org/10.1186/ s12889-018-6250-7

42. Leider JP, Debruin D, Reynolds N, Koch A, Seaberg J. Ethical guidance for disaster response, specifically around crisis standards of care: a systematic review. Am J Public Health. 2017 Sep 1;107(9):e1-9. https://doi. org/10.2105/AJPH.2017.303882

43. Bozzo A. A challenge to unqualified medical confidentiality. J Med Ethics. 2018 Apr 1;44(4):248-52. https://doi.org/10.1136/ medethics-2017-104359

44. George J, Bhila T. Security, confidentiality and privacy in health of healthcare data. Int J Trend Sci Res Develop. 2019 Jun 30;3(4):3737. https://doi.org/10.31142/ijtsrd23780

45. Shahri AB, Ismail Z, Mohanna S. The Impact of the security competency on "self-efficacy in information security" for effective health information security in Iran. J Med Syst. 2016 Nov 1;40(11). https://doi.org/10.1007/s10916016-0591-5 
46. Kruse CS, Smith B, Vanderlinden $H$, Nealand A. Security techniques for the electronic health records. J Med Syst. 2017 Aug 1;41(8). https:// doi.org/10.1007/s10916-017-0778-4

47. Dureab F, Ahmed K, Beiersmann C, Standley CJ, Alwaleedi A, Jahn A. Assessment of electronic disease early warning system for improved disease surveillance and outbreak response in Yemen. BMC Public Health. 2020 Sep 18;20(1422):1-11. https://doi.org/10.1186/ s12889-020-09460-4

48. Brennan B, Gutiérrez V, editores. Guía para la elaboración de la estrategia de comunicación de riesgo: de la teoría a la acción. Washington: Organización Panamericana de la Salud; 2011.

49. Pan American Health Organization, World Health Organization. Communicating about ebola: a guide for leaders [internet]. Ginebra; 2016[citado2021 Apr19].Disponibleen:https:// www.paho.org/hq/dmdocuments/2014/2014cha-communicating-ebola-guide-leaders.pdf

50. Centers for Disease Control and Prevention. Crisis emergency risk comunication [internet]. Vol. 1. Washington DC: Centers for Disease Control and Prevention; 2014 [citado 2021 Apr 19]. Disponible en: https://emergency.cdc. gov/cerc/ppt/cerc_2014edition_Copy.pdf

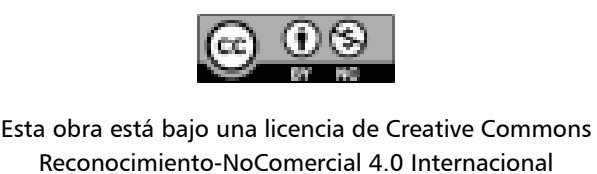

Reconocimiento-NoComercial 4.0 Internacional 